\title{
Heat Kernel Measure on Central Extension of Current Groups in any Dimension
}

\author{
Rémi LÉANDRE
}

Institut de Mathématiques de Bourgogne, Université de Bourgogne, 21000 Dijon, France

E-mail: Remi.leandre@u-bourgogne.fr

Received October 30, 2005, in final form January 13, 2006; Published online January 13, 2006

Original article is available at http://www.emis.de/journals/SIGMA/2006/Paper003/

\begin{abstract}
We define measures on central extension of current groups in any dimension by using infinite dimensional Brownian motion.
\end{abstract}

Key words: Brownian motion; central extension; current groups

2000 Mathematics Subject Classification: 22E65; 60G60

\section{Introduction}

If we consider a smooth loop group, the basical central extension associated to a suitable KacMoody cocycle plays a big role in mathematical physics [3, 11, 21, 24]. Léandre has defined the space of $L^{2}$ functionals on a continuous Kac-Moody group, by using the Brownian bridge measure on the basis [16] and deduced the so-called energy representation of the smooth Kac-Moody group on it. This extends the very well known representation of a loop group of AlbeverioHoegh-Krohn [2].

Etingof-Frenkel [13] and Frenkel-Khesin [14] extend these considerations to the case where the parameter space is two dimensional. They consider a compact Riemannian surface $\Sigma$ and consider the set of smooth maps from $\Sigma$ into a compact simply connected Lie group $G$. We call $C_{r}(\Sigma ; G)$ the space of $C^{r}$ maps from $\Sigma$ into $G$ and $C_{\infty}(\Sigma ; G)$ the space of smooth maps from $\Sigma$ into $G$. They consider the universal cover $\tilde{C}_{\infty}(\Sigma ; G)$ of it and construct a central extension by the Jacobian $J$ of $\Sigma$ of it $\hat{C}_{\infty}(\Sigma ; G)$ (see [7, 8, 25] for related works).

We can repeat this construction if $r>s$ big enough for $C_{r}(\Sigma ; G)$. We get the universal cover $\tilde{C}_{r}(\Sigma ; G)$ and the central extension by the Jacobian $J$ of $\Sigma$ of $\tilde{C}_{r}(\Sigma ; G)$ denoted by $\hat{C}_{r}(\Sigma ; G)$.

By using Airault-Malliavin construction of the Brownian motion on a loop group [1, 9], we have defined in [19] a probability measure on $\tilde{C}_{r}(\Sigma ; J)$, and since the Jacobian is compact, we can define in [19] a probability measure on $\hat{C}_{r}(\Sigma ; G)$.

Maier-Neeb [20] have defined the universal central extension of a current group $C_{\infty}(M ; G)$ where $M$ is any compact manifold. The extension is done by a quotient of a certain space of differential form on $M$ by a lattice. We remark that the Maier-Neeb procedure can be used if we replace this infinite dimensional space of forms by the de Rham cohomology groups $H(M:$ Lie $G)$ of $M$ with values in Lie $G$. Doing this, we get a central extension by a finite dimensional Abelian groups instead of an infinite dimensional Abelian group. On the current group $C_{r}(M ; G)$ of $C^{r}$ maps from $M$ into the considered compact connected Lie group $G$, we use heat-kernel measure deduced from the Airault-Malliavin equation, and since we get a central extension $\hat{C}_{r}(M ; G)$ by a finite dimensional group $Z$, we get a measure on the central extension of the current group. Let us recall that studies of the Brownian motion on infinite dimensional manifold have a long history (see works of Kuo [15], Belopolskaya-Daletskii [6, 12], Baxendale [4, 5], etc.). 
Let us remark that this procedure of getting a random field by adding extra-time is very classical in theoretical physics, in the so called programme of stochastic-quantization of Parisi$\mathrm{Wu}$ [23], which uses an infinite-dimensional Langevin equation. Instead to use here the Langevin equation, we use the more tractable Airault-Malliavin equation, that represents infinite dimensional Brownian motion on a current group.

\section{A measure on the current group in any dimension}

We consider $C_{r}(M ; G)$ endowed with its $C^{r}$ topology. The parameter space $M$ is supposed compact and the Lie group $G$ is supposed compact, simple and simply connected. We consider the set of continuous paths from $[0,1]$ into $C_{r}(M ; G) t \rightarrow g_{t}(\cdot)$, where $S \in M \rightarrow g_{t}(S)$ belongs to $C_{r}(M ; G)$ and $g_{0}(S)=e$. We denote $P\left(C_{r}(M ; G)\right)$ this path space.

Let us consider the Hilbert space $H$ of maps $h$ from $M$ into Lie $G$ defined as follows:

$$
\int_{\Sigma}\left\langle\left(\Delta^{k}+1\right) h, h\right\rangle d S=\|h\|_{H}^{2},
$$

where $\Delta$ is the Laplace Beltrami operator on $M$ and $d S$ the Riemannian element on $M$ endowed with a Riemannian structure.

We consider the Brownian motion $B_{t}(\cdot)$ with values in $H$.

We consider the Airault-Malliavin equation (in Stratonovitch sense):

$$
d g_{t}(S)=g_{t}(S) d B_{t}(S), \quad g_{0}(S)=e .
$$

Let us recall (see [17]):

Theorem 1. If $k$ is enough big, $t \rightarrow\left\{S \rightarrow g_{t}(S)\right\}$ defines a random element of $P\left(C_{r}(M ; G)\right)$.

We denote by $\mu$ the heat-kernel measure $C_{r}(M ; G)$ : it is the law of the $C^{r}$ random field $S \rightarrow g_{1}(S)$. It is in fact a probability law on the connected component of the identity $C_{r}(M ; G)_{e}$ in the current group.

\section{A brief review of Maier-Neeb theory}

Let us consider $\Pi_{2}\left(C_{r}(M ; G)_{e}\right)$ the second fundamental group of the identity in the current group for $r>1$. The Lie algebra of this current group is $C_{r}(M$; Lie $G)$ the space of $C^{r}$ maps from $M$ into the Lie algebra Lie $G$ of $G$ [22]. We introduce the canonical Killing form $k$ on Lie $G$.

$\Omega^{i}(M ; \operatorname{Lie} G)$ denotes the space of $C^{r-1}$ forms of degree $i$ on $M$ with values in Lie $G$. Following [20], we introduce the left-invariant 2-form $\Omega$ on $C_{r}(M ; G)$ with values in the space of forms $Y=\Omega^{1}(M ; \operatorname{Lie} G) / d \Omega^{0}(M ;$ Lie $G)$ which associates

$$
k\left(\eta, d \eta_{1}\right) .
$$

to $\left(\eta, \eta_{1}\right)$, elements of the Lie algebra of the current group.

For that, let us recall that the Lie algebra of the current group is the set of $C^{r}$ maps $\eta$ from the manifold into the Lie algebra of $G$. $d \eta$ is a $C^{r-1}$ 1-form into the Lie algebra of $G$. Therefore $k\left(\eta, d \eta_{1}\right)$ appears as a $C^{r-1} 1$-form with values in the Lie algebra of $G$. Moreover

$$
d k\left(\eta, \eta_{1}\right)=k\left(d \eta, \eta_{1}\right)+k\left(\eta, d \eta_{1}\right) .
$$

This explains the introduction of the quotient in $Y$. Following the terminology of [20], we consider the period map $P_{1}$ which to $\sigma$ belonging to $\Pi_{2}\left(C_{r}(M ; G)_{e}\right)$ associates $\int_{\sigma} \Omega$. Apparently $P_{1}$ takes its values in $Y$, but in fact, the period map takes its values in a lattice $L$ of $H^{1}(M$; Lie $G)$. 
It is defined on $\Pi_{2}\left(C_{r}(M ; G)_{e}\right)$ since $\Omega$ is closed for the de Rham differential on the current group, as it is left-invariant and closed and it is a 2-cocycle in the Lie algebra of the current group [20]. We consider the Abelian group $Z=H^{1}(M ;$ Lie $G) / L$. $Z$ is of finite dimension.

We would like to apply Theorem III.5 of [20]. We remark that the map $P_{2}$ considered as taking its values in $Y / L$ is still equal to 0 when it is considered by taking its values in $H^{1}(M ; \operatorname{Lie} G) / L$.

We deduce the following theorem:

Theorem 2. We get a central extension $\hat{C}_{r}(M ; G)$ by $Z$ of the current group $C_{r}(M ; G)_{e}$ if $r>1$.

Since $Z$ is of finite dimension, we can consider the Haar measure on $Z$. We deduce from $\mu$ a measure $\hat{\mu}$ on $\hat{C}_{r}(M ; G)$.

Remark 1. Instead of considering $C_{r}(M ; \operatorname{Lie} G)$, we can consider $W_{\theta, p}(M ; \operatorname{Lie} G)$, some convenient Sobolev-Slobodetsky spaces of maps from $M$ into Lie $G$. We can deduce a central extension $\hat{C}_{\theta, p}(M ; G)$ of the Sobolev-Slobodetsky current group $C_{\theta, p}(M ; G)_{e}$. This will give us an example of Brzezniak-Elworthy theory, which works for the construction of diffusion processes on infinite-dimensional manifolds modelled on M-2 Banach spaces, since Sobolev-Slobodesty spaces are M-2 Banach spaces $[9,10,18]$. We consider a Brownian motion $B_{t}^{1}$ with values in the finite dimensional Lie algebra of $Z$ and $\hat{B}_{t}=\left(B_{t}(\cdot), B_{t}^{1}\right)$ where $B_{t}(\cdot)$ is the Brownian motion in $H$ considered in the Section 2. Then, following the ideas of Brzezniak-Elworthy, we can consider the stochastic differential equation on $\hat{C}_{\theta, p}(M ; G)$ (in Stratonovitch sense):

$$
d \hat{g}_{t}(\cdot)=\hat{g}_{t}(\cdot) d \hat{B}_{t}
$$

\section{Acknowledgements}

The Author thanks Professor K.H. Neeb for helpful discussions.

[1] Airault H., Malliavin P., Integration on loop groups. II. Heat equation for the Wiener measure, J. Funct. Anal., 1992, V.104, 71-109.

[2] Albeverio S., Hoegh-Krohn R., The energy representation of Sobolev Lie groups, Compos. Math., 1978, V.36, 37-52.

[3] Araki H., Bogoliubov automorphisms and Fock representations of canonical anticommutation relations, in Operator Algebras and Mathematical Physics, Editors P.A.T. Jorgensen and P.S. Muhly, Contemp. Math., 1987, V.62, 23-143.

[4] Baxendale P., Markov processes on manifolds of maps, Bull. Amer. Math. Soc., 1976, V.82, 505-507.

[5] Baxendale P., Wiener processes on manifolds of maps, Proc. Roy. Soc. Edinburgh Sect. A, 1980/1981, V.87, $127-152$.

[6] Belopolskaya Y.L., Daletskii Yu.L., Stochastic differential equation and differential geometry, Kluwer, 1990.

[7] Berman S., Billig Y., Irreducible representations for toroidal Lie algebras, J. Algebra, 1999, V.221, $188-231$.

[8] Berman S., Gao Y., Krylyuk Y., Quantum tori and the structure of elliptic quasi-simple algebras, J. Funct. Anal., 1996, V.135, 339-389.

[9] Brzezniak Z., Elworthy K.D., Stochastic differential equations on Banach manifolds, Methods Funct. Anal. Topology, 2000, V.6, 43-80.

[10] Brzezniak Z., Léandre R., Horizontal lift of an infinite dimensional diffusion, Potential Anal., 2000, V.12, 43-84.

[11] Carey A.L., Ruijsenaars S.N.M., On fermion gauge groups, current algebras and Kac-Moody algebras, Acta Appl. Math., 1987, V.10, 1-86.

[12] Daletskii Yu.L., Measures and stochastic differential equations on infinite-dimensional manifolds, in Espace de lacets, Editors R. Léandre, S. Paycha and T. Wuerzbacher, Publication University of Strasbourg, 1996, 45-52.

[13] Etingof P., Frenkel I., Central extensions of current groups in two dimension, Comm. Math. Phys., 1994, V.165, 429-444. 
[14] Frenkel I., Khesin B., Four dimensional realization of two-dimensional current groups, Comm. Math. Phys., 1996, V.178, 541-562.

[15] Kuo H.H., Diffusion and Brownian motion on infinite dimensional manifolds, Trans. Amer. Math. Soc., 1972, V.159, 439-451.

[16] Léandre R., A unitary representation of the basical central extension of a loop group, Nagoya Math. J., 2000, V.159, 113-124.

[17] Léandre R., Brownian surfaces with boundary and Deligne cohomology, Rep. Math. Phys., 2003, V.52, 353-362.

[18] Léandre R., Galton-Watson tree and branching loops, in Geometry, Integrability and Quantization. VI (Softek), Editors A. Hirshfeld and I. Mladenov, 2005, 276-283.

[19] Léandre R., Measures on central extension of current groups in two dimension, in Infinite Dimensional Analysis (Jagna), Editors C. Bernido and V. Bernido, to appear.

[20] Maier P., Neeb K.H., Central extensions of current group, Math. Ann., 2003, V.326, 367-415.

[21] Mickelsson J., Current algebras and groups, Plenum Press, 1989.

[22] Milnor J., Remarks on infinite-dimensional Lie groups, in Relativity, Groups and Topology II, Editor B. Dewitt, North-Holland, 1984, 1007-1057.

[23] Paris G., Wu Y.S., Perturbation theory without gauge fixing, Sci. Sinica, 1981, V.24, 483-496.

[24] Pressley A., Segal G., Loop groups, Oxford University Press, 1986.

[25] Zhiyang S., Toroidal groups, Comm. Algebra, 1992, V.20, 3411-3458. 